\title{
Atmospheric propagation of high power laser radiation at different weather conditions
}

\author{
Carsten Pargmann*a, Thomas Hall ${ }^{\mathrm{b}}$, Frank Duschek ${ }^{\mathrm{a}}$, Jürgen Handke ${ }^{\mathrm{a}}$ \\ ${ }^{a}$ German Aerospace Center (DLR), Institute of Technical Physics, Langer Grund, \\ D-74239 Hardthausen, Germany; \\ ${ }^{\mathrm{b}}$ German Aerospace Center (DLR), Institute of Technical Physics, Pfaffenwaldring 38-40, \\ D-70569 Stuttgart, Germany
}

\begin{abstract}
Applications based on the propagation of high power laser radiation through the atmosphere are limited in range and effect, due to weather dependent beam wandering, beam deterioration, and scattering processes. Security and defense related application examples are countermeasures against hostile projectiles and the powering of satellites and aircrafts. For an examination of the correlations between weather condition and laser beam characteristics DLR operates at Lampoldshausen a $130 \mathrm{~m}$ long free transmission laser test range. Sensors around this test range continuously monitor turbulence strength, visibility, precipitation, temperature, and wind speed. High power laser radiation is obtained by a TruDisk 6001 disk laser (Trumpf company) yielding a maximum output power of $6 \mathrm{~kW}$ at a wavelength of $1030 \mathrm{~nm}$. The laser beam is expanded to $180 \mathrm{~mm}$ and focused along the beam path. Power and intensity distribution are measured before and after propagation, providing information about the atmospheric transmission and alterations of diameter and position of the laser beam. Backscattered laser light is acquired by a photo receiver. As a result, measurements performed at different weather conditions show a couple of correlations to the characteristics of the laser beam. The experimental results are compared to a numerical analysis. The calculations are based on the Maxwell wave equation in Fresnel approximation. The turbulence is considered by the introduction of phase screens and the "von Karman" spectrum.
\end{abstract}

Keywords: high energy laser, atmospheric propagation, disk laser, laser beam characterization

\section{INTRODUCTION}

In more and more applications high energy laser radiation is leaving the controllable conditions of a laboratory and is propagated outside through the air. Power beaming of unmanned vehicles and satellites, countermeasures against hostile $\operatorname{targets}^{1}$ as well as tracking of space debris built up examples in the domain of defense and security. However, the atmospheric condition influences the propagation of the laser beam ${ }^{2,3}$. The resulting effect is accumulated with distance and has to be taken into account. Beam wandering and beam deterioration lead to reduced power densities at the target and limit the range of operation. Furthermore, a redirection of a part of the energy to unwanted directions, summarized here as scattering processes, reduces the power transmission and is a matter of laser safety, both for nearby civilians and for military units.

For the experimental analysis of the dependence of the beam propagation on the weather condition the DLR operates at Lampoldshausen a free transmission laser test range. It consists of two stations, the transmitting (TS) and the receiving (RS) one, which enclosure a $130 \mathrm{~m}$ long pathway with a beam path $1 \mathrm{~m}$ above asphalt ground. Several sensors around the test range continuously monitor the atmosphere, at all weather conditions. The simultaneous characterization of the laser beam is performed with sensors inside of the TS and RS. These measurements are corresponding to power, shape and jitter of the main beam as well as to the beam portion, which is scattered apart.

For a better classification and understanding of the measurement results and in order to be able to extrapolate the experiments to different propagation scenarios the atmospheric laser beam propagation is analyzed numerically. This analysis is performed by the software TALAP (Turbulent Atmosphere and Laser Beam Propagation), developed at the Institute of Technical Physics of DLR.

*carsten.pargmann@dlr.de; phone +49 6298 28-586; fax +49 6298 28-582; www.dlr.de/tp 

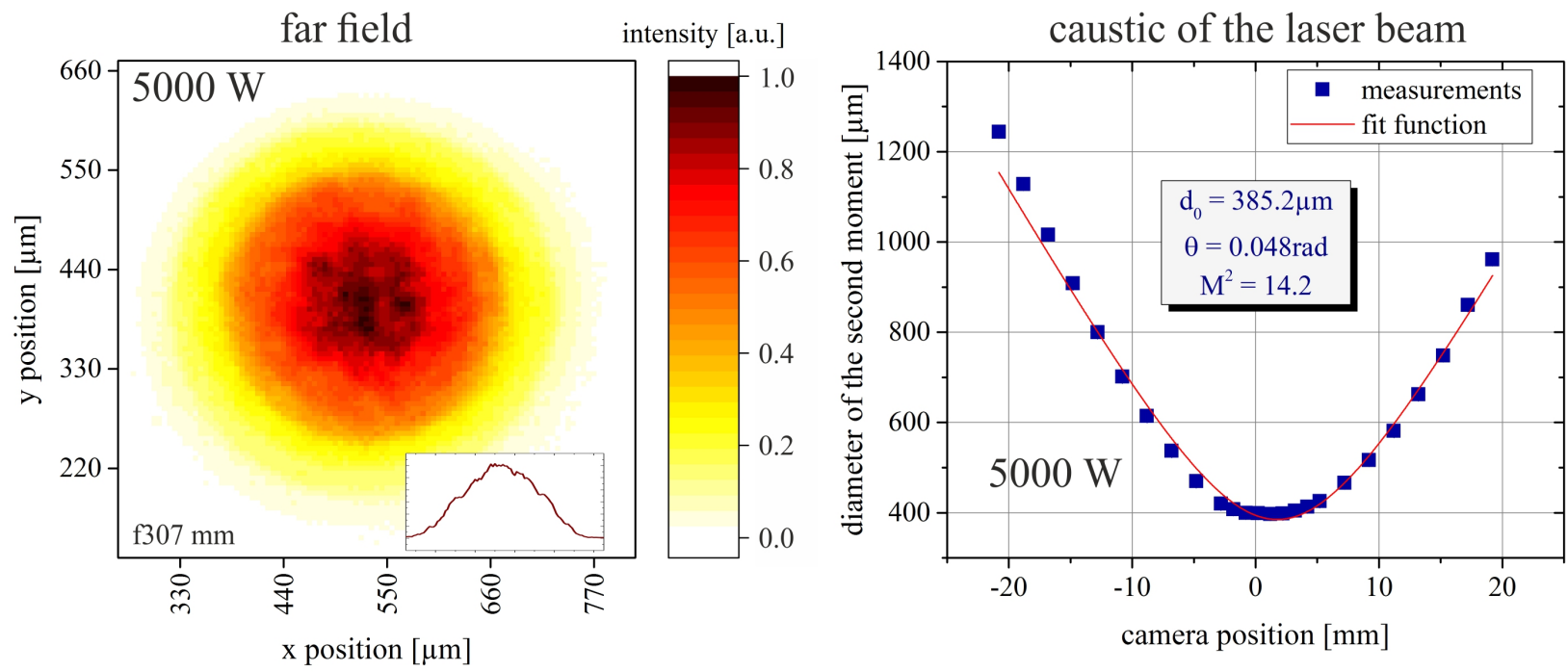

Figure 1. Far field intensity distribution in the focus of a lens with a focal length of $307 \mathrm{~mm}$ (left) and the change of the second moment diameter with the change of the camera position (right).

\section{LASER SYSTEM AND TEST RANGE}

Nowadays, solid state high power lasers reach power levels of $10 \mathrm{~kW}$ and above with noteworthy beam qualities ${ }^{4,5}$. The laser system used here is a TruDisk 6001 (4C) disk laser (Trumpf Company), consisting of two cavities. They operate at a wavelength of $1.03 \mu \mathrm{m}$ and achieve an adjustable output power (continuous wave) between $180 \mathrm{~W}$ and $6000 \mathrm{~W}$. The laser radiation is coupled into a $40 \mathrm{~m}$ long optical fiber with a core diameter of $100 \mu \mathrm{m}$ guiding the beam into the TS of the free transmission laser test range.

The beam quality of the disk laser system is obtained by measuring the second moment diameters of intensity distributions in the TS along a caustic near the focus point of a lens with a focal length of $307 \mathrm{~mm}$. In Figure 1 the intensity distribution of the far field and the caustic of the laser beam at an output power of $5000 \mathrm{~W}$ are shown. The measurements are performed using a beam diagnostic camera Beamage-3.0 by Gentec-EO Inc. positioned on a translation stage. As a result the beam propagation factor $\mathrm{M}^{2}$ is obtained to be 14.2.

For a comparison between experimental measurements and numerical simulations the intensity and wavefront distribution of the near field is measured right after the collimation unit at the exit of the optical fiber in the $\mathrm{TS}^{6}$. The measurements are performed using the wavefront sensor SID4 (Phasics Corp.). As a result, at an output power of $5000 \mathrm{~W}$ the diameter of the laser beam at the output of the collimation unit is about $18.3 \mathrm{~mm}$. The measured distributions are used as initial distributions for the numerical analysis.

For the analysis of the influence of the weather condition on the beam propagation in air the beam of the disk laser is propagating along a $130 \mathrm{~m}$ long pathway from the transmitting (TS) to the receiving station (RS), $1 \mathrm{~m}$ above asphalt ground. A sketch of the free transmission laser test range is shown in Figure 2. It is surrounded by earth walls protecting the region outside the test range against direct or indirect laser radiation. Figure 2 also shows the locations of most important sensors characterizing the current weather condition.

A scintillometer measures the strength of the optical turbulences in the air, the refractive index structure parameter $\mathrm{Cn}^{2}$. The turbulence measurement crosses the laser beam path and is also positioned $1 \mathrm{~m}$ above the ground. The precipitation is measured by a disdrometer. The system is able to differentiate between different kinds of precipitation (rain, snow, hail, sleet) by measuring the diameter and velocity distributions of the droplets. Near the center of the test range the visibility and the wind speed and direction are measured. Various temperature sensors are positioned in and at different heights above the ground. Pressure and relative humidity of the air are monitored and an aerosol spectrometer gives additional information about the size distribution of aerosol particles. 
Propagation measurements are performed at different atmospheric conditions. These include diverse types of rain, fog, haze, snow, dust and other conditions. On the laser test range sensors monitoring the weather conditions operate continuously night and day, independent on work or research activities.

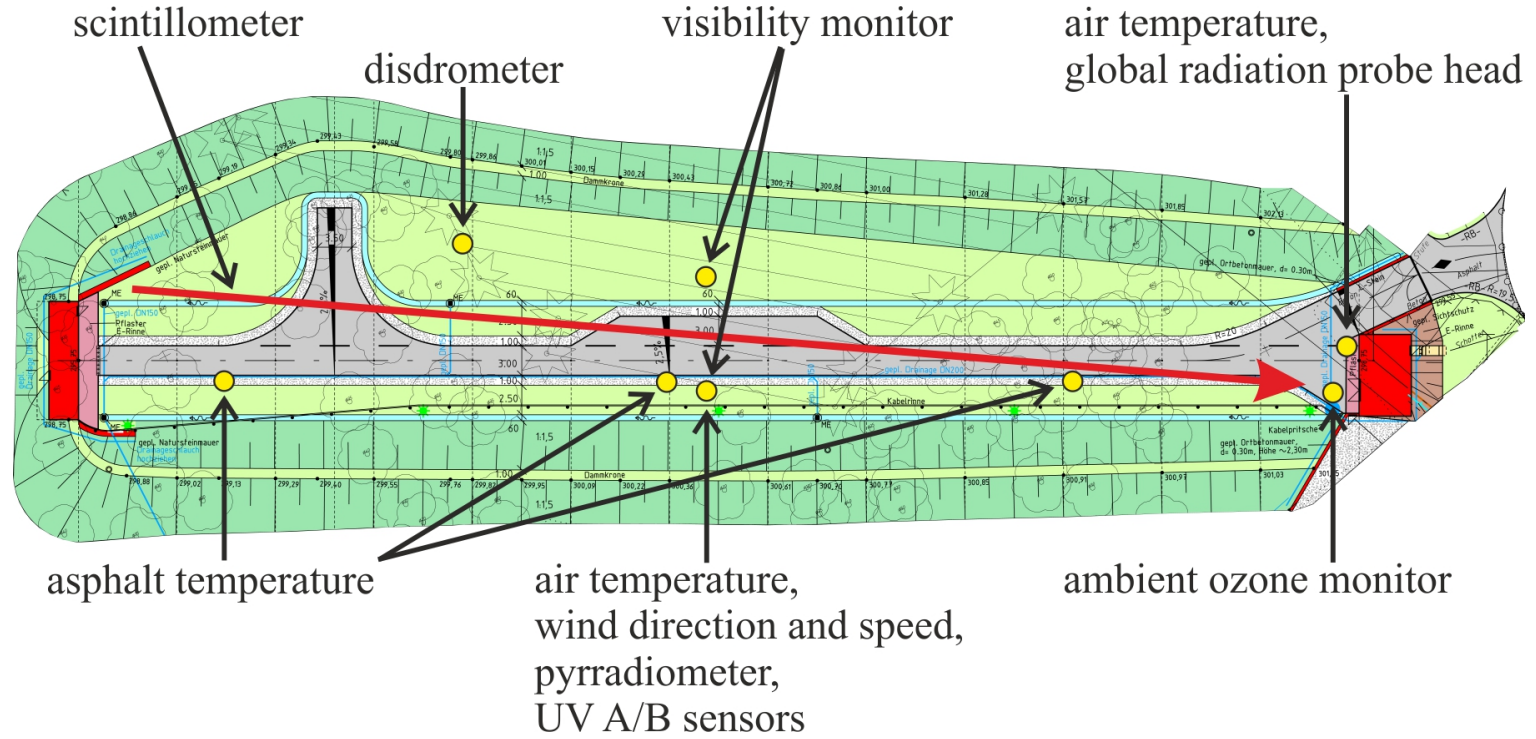

Figure 2. Sketch of the free transmission laser test range and location of important sensors (top view).

\section{OPTICAL SETUP}

The radiation of the disk laser is coupled into a $40 \mathrm{~m}$ long optical fiber, guiding the laser beam to an optical setup inside of the TS (shown in Figure 3) and at first to the entrance of a collimation unit. The collimated beam is guided into a telescope setup consisting of two off-axis parabolic mirrors with respective focal lengths of $227 \mathrm{~mm}$ and $2272 \mathrm{~mm}$, magnifying the beam to a diameter of approximately $180 \mathrm{~mm}$. The distance of the telescope mirrors is adjusted to obtain the focus point in $135 \mathrm{~m}$ distance. Two computer controlled direct-drive rotary stages bear the transmitting mirror and guide the laser radiation out of the TS and further to the direction of the RS at the end of the test range. A part of the laser beam is beforehand reflected out of the main path to the diagnostic setup. A thermopile measures the integrated power during the complete measurement time. A photodetector coupled to an Ulbricht sphere is calibrated with the help of the thermopile and measures fast changes of the laser output power. A beam diagnostic camera (Beamage-3.0 from Gentec-EO Inc.) measures the intensity distribution of the laser beam and calculates the effective beam diameter containing $86 \%$ of the overall power as well as the position of the center of gravity of the distribution. The camera is recording at a speed of 10 frames per second. The exposure time is below $1.0 \mathrm{~ms}$.

The optical diagnostic setup inside of the RS is also shown in Figure 3. Basically, it consists of the same elements as inside of the TS. The main difference is constituted by the measurement of the intensity distribution. Instead of guiding the laser beam directly onto the camera, an optical wedge with a free aperture of $140 \mathrm{~mm}$ reflects a part of the laser beam onto a reflective diffusing screen consisting of sand blasted aluminum. The camera (same model as the one used in the TS) images the intensity distribution directly from the screen. The main path of the laser beam is terminated by an absorber.

A part of the laser radiation is expected to be scattered by the atmosphere to all directions. In order to obtain a measure for the amount of radiation which is scattered back from the test range to the direction of the TS, an off-axis parabolic mirror is positioned $0.8 \mathrm{~m}$ away from the main beam inside of the TS. The optical axis of the mirror is aligned to collect scattered radiation from an average distance of $20 \mathrm{~m}$ away from the TS onto a photo receiver (Femto OE-200-SI). The detection volume is constituted by the intersection of the laser beam and the line-of-sight of the detection mirror. An aperture on the test range blocks the back scattered radiation of the optical setup inside of the RS. 

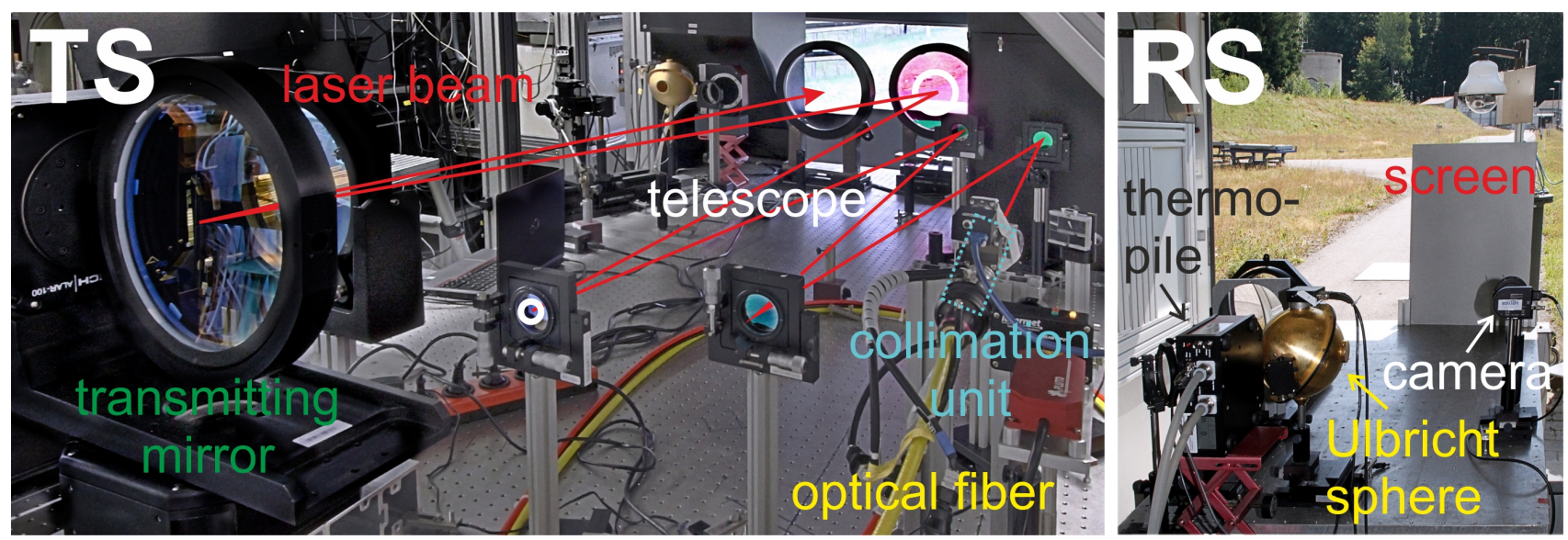

Figure 3. Optical setup inside of the transmitting (TS) and the receiving station (RS).

\section{NUMERICAL SIMULATION}

The turbulent motion of the air caused by convection and wind-velocities induces random fluctuations in the refractive index of the atmosphere. Although these variations of the refractive index are very low, a large propagation distance of a laser beam through such a medium generates a considerable effect to the optical quality of the beam.

The Kolmogorov turbulence model states that atmospheric temperature variations and wind-speed fluctuations create local unstable air masses. These consist of turbulent cells of different scale sizes. The energy, determining the turbulence is fed into the large scale size cell and then cascades down at a constant rate per unit mass to small scales where it is dissipated by molecular viscosity.

The numerical analysis of the laser beam propagation is performed by the software TALAP (Turbulent Atmosphere and Laser Beam Propagation), developed at the Institute of Technical Physics of DLR. In doing so, the refractive-index fluctuations due to turbulent flow fields are characterized by the von Karman power spectrum

$$
\Phi_{n}=0.033 \cdot C_{n}^{2} \cdot\left(k^{2}+k_{0}^{2}\right)-\frac{11}{6} \cdot e^{-\frac{k^{2}}{k_{m}^{2}}}
$$

where $\mathrm{C}_{\mathrm{n}}^{2}$ is the refractive index structure constant in $\left[\mathrm{m}^{-2 / 3}\right]$, $\mathrm{k}$ is the spatial wave number, $\mathrm{k}_{0}=2 \pi / \mathrm{L}_{0}$ and $\mathrm{k}_{\mathrm{m}}=5.92 / \mathrm{l}_{0}$. $\mathrm{L}_{0}$ and $\mathrm{l}_{0}$ describe the outer and inner scale in the meter $\left(\mathrm{L}_{0}\right)$ and millimeter range $\left(\mathrm{l}_{0}\right)$.

In the turbulence phase-screen approach, the propagation distance is divided into a number of segments and the cumulative phase shift of any segment is considered to lie in a plane. A random function describes the fluctuations of the refractive index. For the numerical evaluation of the phase shift a standard $\operatorname{method}^{7}$ is used.

For the numerical simulation a Fast Fourier transform (FFT) method is applied. The computational region has an area of $50 \mathrm{~cm} \times 50 \mathrm{~cm}$ with a grid sampling rate of $2^{11}$ in each dimension. The propagation distance is divided into 20 segments with equally spaced phase screens. Experimentally obtained values are used for the parameters $\mathrm{C}_{\mathrm{n}}{ }^{2}$ and $\mathrm{l}_{0}$. The magnitude of $\mathrm{L}_{0}$ is selected in such a way, that it is approximately suitable for the height of the laser beam above asphalt ground. The computation time to simulate one propagation distance between transmitting and receiving station amounts to about 2 minutes on a standard workstation computer (6 CPU cores and 24 GB RAM).

\section{RESULTS}

First measurements of the laser beam parameters have been performed at different atmospheric conditions. Exemplary in Figure 4 the turbulence strength and the jitter of the laser beam are shown in the course of a typical summer day. The output power of the laser is $5000 \mathrm{~W}$. The turbulence strength is measured by the scintillometer along a time period of one minute. The jitter of the laser beam is measured inside of the TS as well as inside of the RS. The jitter is the standard deviation of the center of gravity of the intensity distribution. Each deviation is measured in horizontal and vertical direction and is obtained from 100 frames in $10 \mathrm{~s}$ of measurement time. The right graph in Figure 4 is obtained by 
subtracting the beam jitter measured inside the TS from the beam jitter inside the RS. Beforehand the jitter measured in the TS is corrected by the ratio of the focal lengths.
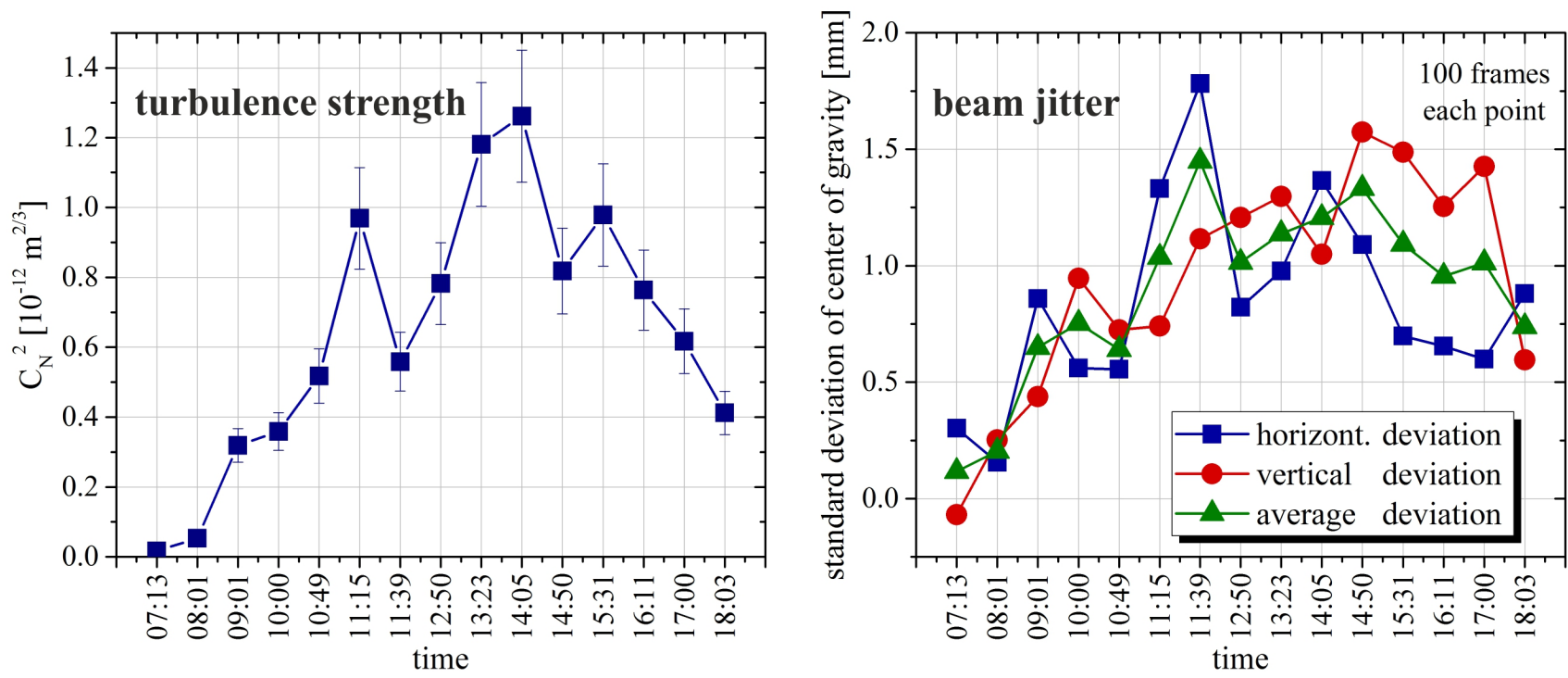

Figure 4. Turbulence strength (left) and beam jitter (right) in the course of a typical day in central European summer.

The green triangles show the average of the horizontal and the vertical beam jitter. According to the difference in horizontal and vertical deviation the error bars for the average beam jitter are calculated to be around $33 \%$. On first sight the beam jitter is approximately following the turbulence strength in the course of the day. But there are some differences. For instance, at 11:39 the turbulence strength shows a dip whereas the beam jitter shows a peak. One explanation for this difference is offered by the error bars, but another reason is given by the different measurement time periods.

For the numerical simulations the best fit of a Gaussian fit function of the measured intensity distribution of the near field is used as starting point for the far field simulations. The beam propagation factor $\mathrm{M}^{2}$ is simulated by a defocusing phase in the near field. Thus the numerically obtained beam diameter without consideration of turbulence correlates well with the experimentally expected value.
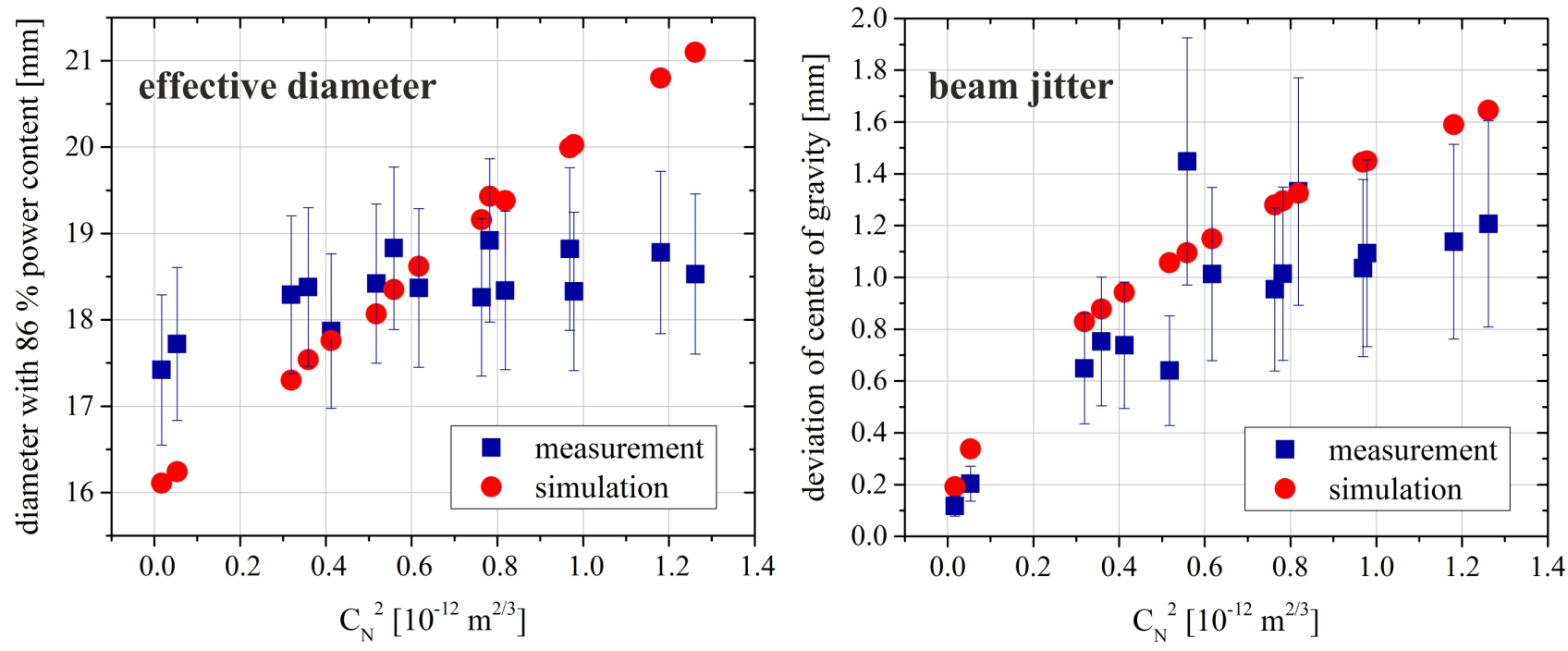

Figure 5. Effective diameter (left) and beam jitter (right) in experiment and simulation. 
In Figure 5 a comparison between experiment and simulation of the effective diameter and the beam jitter in dependence on the turbulence strength is shown. The measurements of the effective diameter containing $86 \%$ of the total power show a low dependence on the considered turbulence strengths, whereas the simulation shows a change of up to $30 \%$. However, the comparison of the beam jitter shows a very promising correspondence. In the near future intensity distribution measurements in the RS will be performed using a movable reflective diffusing screen. The reduction of speckles may further help to improve the results.

Stray light measurements have been performed at different precipitation conditions. On the left hand side of Figure 6 the power transmission loss between the TS and the RS in dependence on the stray light power measured in the TS is shown. The output power is $5000 \mathrm{~W}$ and each measurement point is obtained during a time interval of one minute. The measurements were performed on two different days. One day showed a precipitation consisting of a mixture of snowflakes and rain and on the other day only large snowflakes were falling down. The standard deviation of the stray light measurement points are caused by the inhomogeneous temporal distribution of the snowflakes. The graph demonstrates the correlation between the power loss of the main beam and its redistribution to other directions. On the right hand side of Figure 6 the dependence of the stray light power on the amount of snow is shown. These measurements were performed on the same days as before. The amount of snow is measured by the disdrometer. Snowfall is recognized due to the velocity and diameter distribution of the droplets. In the case of snowfall the given precipitation intensity is a recalculation using the liquid water equivalent. The error bars correspond to a standard deviation of $30 \%$. The measurement points indicate a dependence of the intensity of the stray light on the kind of precipitation. Same amounts of snow result to different power levels measured by the photo receiver. The values next to the encircled measurement points represent the cross-section of the droplets seen by the disdrometer during a time range of 3 minutes. Hence, a droplet distribution with the same total volume but increased cross-section experiences more scattering, or reflection, apart from the main laser beam. Further measurements at different droplet diameter distributions will have to verify and if applicable to quantify this indication.
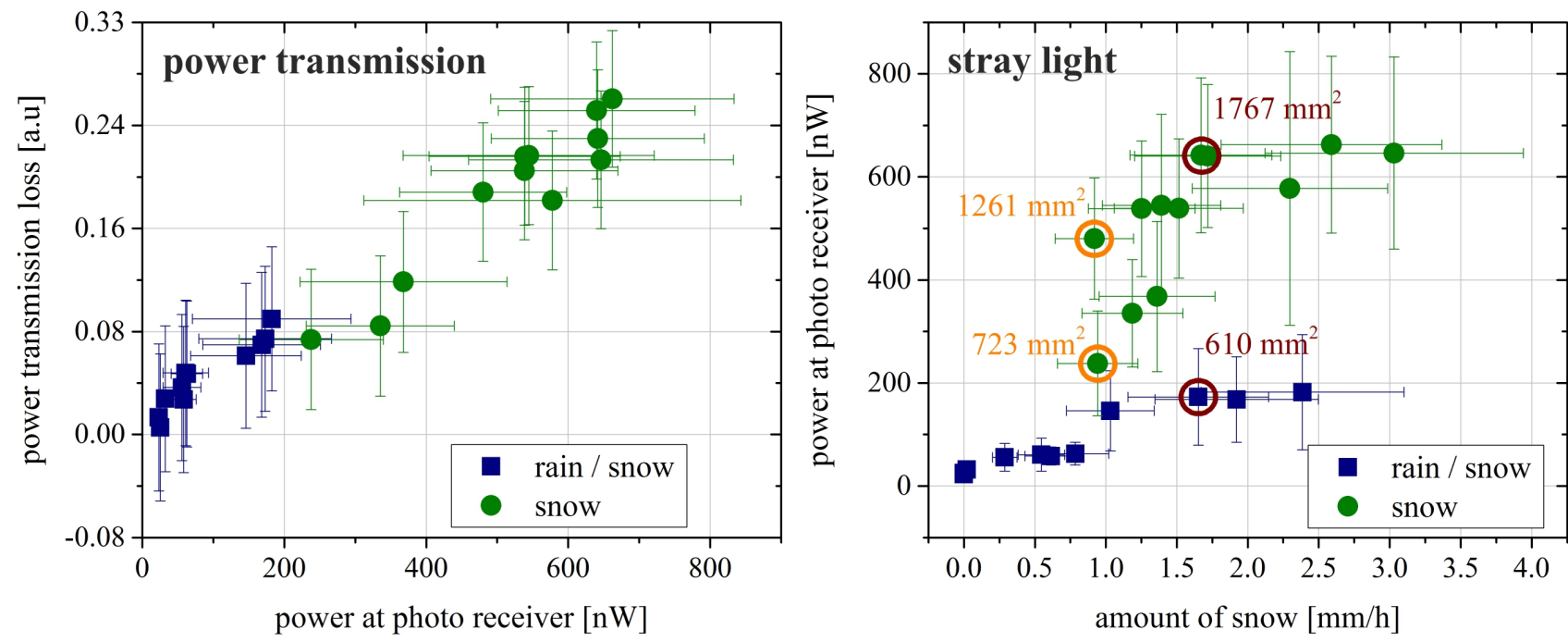

Figure 6. Power transmission loss in dependence on the measured stray light (left) and power at photo receiver in dependence on the amount of snow (right). The numbers denote the cross-section area of all droplets in a time range of 3 minutes.

\section{SUMMARY AND OUTLOOK}

Simultaneous measurements of the atmospheric condition and the optical parameters of a high power laser beam before and after the propagation along a $130 \mathrm{~m}$ free transmission laser test range are demonstrated. Measurements of the beam jitter, beam diameter, power transmission and power of stray light are shown. Numerical simulations of the laser propagation show promising results, especially regarding the beam jitter. Stray light measurements have been performed at different precipitation conditions.

However, more data with comprehensive statistics are crucial in order to get a better insight into the atmospheric propagation of high power laser radiation. Hence, future measurements will be performed more automatically and will 
cover further atmospheric conditions and parameters. In order to improve the comparison results the optical measurement setup and the numerical program will be optimized and extended, experimentally for instance by the use of a movable reflective diffusing screen in the RS.

The measurement of the stray light shows an interesting dependence on the kind of precipitation. A further examination should also include stray light measurements under different angles. In addition a comparison with stray light theory will follow up.

\section{REFERENCES}

[1] Fiorino, S., Bartell, R. J., Krizo, M. J., and Cusumano, S. J., "Propagation variability assessments of ship defense HEL and HPM performance in worldwide maritime boundary layer environments at wavelengths of $1.0642 \mu \mathrm{m}, 2.141 \mu \mathrm{m}, 3.16 \mathrm{~mm}$ and $12.2 \mathrm{~cm}$," Proc. SPIE 6951, 69510G (2008).

[2] Weichel, H., “Atmospheric propagation of laser-beams," Proc. SPIE 547, 1-15 (1985).

[3] Sprangle, P., Ting, A., Penano, J., Fischer, R., and Hafizi, B., "Incoherent combining and atmospheric propagation of high-power fiber lasers for directed-energy applications," IEEE J. Quant. Electron. 45(1-2), 138148 (2009).

[4] Giesen, A., Hügel, H., Voss, A., Wittig, K., Brauch, U., and Opower, H., "Scalable concept for diode-pumped high-power solid-state lasers, “ Appl. Phys. B 58(5), 365-372 (1994).

[5] Limpert, J., Röser, F., Klingebiel, S., Schreiber, T., Wirth, C., Peschel, T., Eberhardt, R., and Tünnermann, A., „The rising power of fiber lasers and amplifiers,“ IEEE J. Sel. Top. Quantum Electron. 13(3), 537-545 (2007).

[6] Pargmann, C., Hall, T., Duschek, F., Fischbach, T., Grünewald, K. M., Klaffki, K., Thieser, and J., Handke, J., „Experimental and numerical analysis of atmospheric propagation of high energy laser,“ Proc. SPIE 9650, 96500L (2015).

[7] Fleck Jr., J. A., Morris, J. R., and Feit, M. D., “Time-dependent propagation of high energy laser beams through the atmosphere," Appl. Phys. 10(2), 129-160 (1976). 